\title{
Modeling neutral beam transport in fusion experiments: Studying the effects of reionisation and deflection
}

\author{
Niek den Harder, Guillermo Orozco, Irene Zammuto, Christian Hopf, the ASDEX Upgrade Team
}

\author{
Max-Planck-Institut für Plasmaphysik, Garching bei München, Germany
}

\begin{abstract}
A crucial component for any Neutral Beam Injection system is the beam duct, which connects the beam sources to the fusion reactor. There are two types of losses in the duct: geometrical losses due to scraping of the beam on beamline components, and reionisation losses due to interaction of the beam with background gas. A suite of modeling tools has been created to get insight in the magnitude of the losses and power deposition on beamline components: a molecular flow code taking into account the full 3D geometry to calculate background gas densities, FEM calculations in ANSYS to calculate magnetic fields, particle tracking through the magnetic field while interacting with the background gas via a Monte-Carlo scheme to calculate beam losses and resulting power loads. To illustrate the potential of the suite of models, the NBI beamline 2 of the ASDEX Upgrade tokamak is studied, which recently underwent an upgrade of the box exit scraper. Gas densities and magnetic fields are calculated and compared with measurements. Calculated heat loads are benchmarked with the calorimetry on the residual ion dumps. Power deposition in the duct is calculated before and after the installation of the new NBI box exit scraper to assess the impact of this upgrade.
\end{abstract}

Keywords: NBI, Modeling, Duct losses, Heat loads, Reionisation, Computer Aided Design

\section{Introduction}

Neutral beam injection has been successful in producing high fusion performance plasmas in all of the large tokamaks operating worldwide [1]. A crucial component for any NBI system is the beam duct, which connects the beam sources to the fusion reactor vessel. Especially for NBI beamlines intended for high-power long-pulse operation, it is important for machine safety that the losses are well quantified, so that adequate cooling and protection of beamline components can be implemented. A good understanding of the losses could help minimize them and improve the energy efficiency of the beamline.

There are two main types of beamline losses, geometrical losses, due to scraping of the beam on beamline components, and reionisation losses, due to interaction of the beam with background gas. Geometrical losses and the resulting heat loads are straightforward to calculate when the beamline and source geometry is known. In contrast, quantifying reionisation losses requires knowledge of the gas density in the beamline, and the resulting heat deposition depends on the local magnetic field. Because of the deflection of the reionized particles, heat deposition may occur in shadowed, distant, and unexpected locations. The physics of both types of losses is well understood, and lends itself well to modeling.

A suite of tools is created which model gas flow, magnetic field, and particle transport. With these tools, it is possible to calculate geometrical losses as well as reionisation losses, and the resulting heat deposition for any beamline with minimal lead time, which enables improvement of the beamline in an iterative process. As an illustration of the capabilities of the codes, the NBI beamline 2 of the ASDEX Upgrade tokamak is studied.

In NBI beamline 2 of ASDEX Upgrade, the D800 gate valve connecting the beam box to the duct has been damaged several times in the past. Previous research has shown that the damaged locations are shadowed, so that the damage could only be caused by reionized particles [2]. As preventive measure, a new, more restrictive scraper at the exit of the NBI vacuum vessel, the Box Exit Scraper (BES), has been installed before the 2017 experimental campaign. The developed codes are used to quantify the impact of the new BES on the power losses and heat deposition in the duct. The neutral background gas density is calculated for several pumping speeds, and benchmarked with pressure measurements. The magnetic field is calculated with finite element calculations in ANSYS for several time points in various discharges. Heat loads on components are calculated. The effects of reionisation and subsequent deflection are studied for the old and new scraper geometry.

Section 2 introduces the experiment for which calculations were performed by describing the beam generation, beam transport, and vacuum system. In Section 3 the computational methods are presented which calculate the neutral gas density, magnetic field, and beam propagation with background gas interaction. Section 4 shows the calculated neutral gas densities in comparison with pressure measurements, the calculated heat loads on the residual ion dumps together with the measured calorimetry, the transmission losses in the beam duct, and the resulting power density.

\section{Experimental layout}

At the ASDEX Upgrade tokamak, NBI delivers up to 20 MW, representing more than half of the total installed 
heating power. There are two neutral beam boxes, each with 4 positive ion sources, that inject a beam power of $2.5 \mathrm{MW}$ per source. In beam box 1 , ions are extracted from an arc plasma ion source over a potential drop of $60 \mathrm{kV}$. Beam box 2 operates at an acceleration voltage of $93 \mathrm{kV}$, with RF-sources [3].

The $\mathrm{D}^{+}, \mathrm{D}_{2}{ }^{+}$, and $\mathrm{D}_{3}{ }^{+}$ions are extracted by a set of 3 grids with 774 apertures, where the third grid prevents back-acceleration of negative ions [4]. Each grid is divided into two grid halves that are tilted vertically by $0.87^{\circ}$ to focus the beam. In addition, the apertures in the plasma facing grid have an offset that increases linearly with distance from the grid half center, to induce an inward steering of the outer beamlets. Directly after the source the extracted ions interact with $\mathrm{D}_{2}$ background gas in a tapered pipe, the neutralizer, leading to dissociation of the molecular ions and neutralization. In addition, background gas interaction leads to excitation and light emission. The Balmer- $\alpha$ emission is used to diagnose the species fractions and beam divergence [5]. The non-neutralized beam is deflected by approximately $180^{\circ}$ on the residual ion dump, which is equipped with 6 actively cooled panels arranged in a V-shape, each capable of absorbing $570 \mathrm{~kW}$ with a peak power density of $25 \mathrm{MW} / \mathrm{m}^{2}$ in steady state [6]. Minority species such as $\mathrm{D}^{-}$and $\mathrm{D}_{2}^{+}$are dumped on dedicated panels. A cutaway CAD model of the beam box is shown in Figure 1.

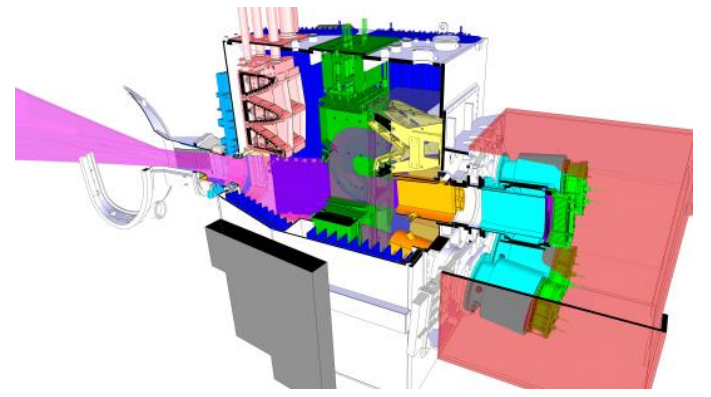

Fig. 1. Cutaway drawing of the beam box. Ions are extracted from an RF-plasma (green), interact with background gas in the neutralizer (cyan + orange). Residual ions are deflected by the deflection magnet (green) and dumped on the residual ion dumps (yellow). The titanium getter pumps are dark blue.

The NBI beam box is connected to the duct with a gate valve. The box exit scraper protects this sensitive vacuum component from the beams. The old BES, which was used before 2017, is D-shaped. It completely protects the gate valve from particles coming directly from the source. However, since the gate valve behind it was damaged on the right side, it was speculated that deflected reionized particles still reached these parts of the gate valve. The new BES is symmetric, and extends further in the duct to intersect these deflected trajectories. Both scrapers are shown together with the gate valve in Figure 2.

Each NBI box has a volume of approximately $30 \mathrm{~m}^{3}$, divided into two chambers, which are separated by the residual ion bending magnet and an additional vacuum shield [7]. The base pumping system consists of two TPH 2200 turbomolecular pumps with a total pumping speed of $6 \mathrm{~m}^{3} \mathrm{~s}^{-1}$ at a pressure of $10^{-4} \mathrm{mbar}$ for deuterium [8]. They pump down the box to a pressure of $10^{-5}$ to $10^{-6}$ mbar, depending on the leak rate. During a beam pulse with all four sources, the gas inlet is $20 \mathrm{~Pa} \mathrm{~m}^{3} \mathrm{~s}^{-1}$. To keep the pressure down during a beam pulse, 2 titanium getter pumps are installed per chamber with a total maximum pumping speed of $3000 \mathrm{~m}^{3} \mathrm{~s}^{-1}$ for hydrogen [7]. The large pumping speed is achieved by using almost the complete side walls of the box, which is 4 meters high. Corrugated deposition panels further increase the surface area of the pumps.

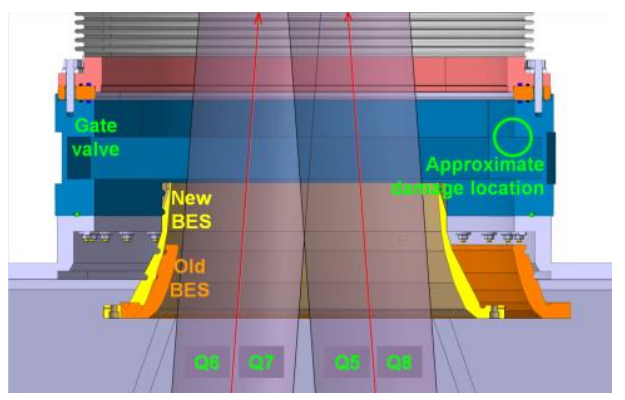

Fig. 2. Horizontal cut through the old and new BES which protect the gate valve.

\section{Computational methods}

\subsection{Neutral gas density calculation}

In the NBI box, the pressure is sufficiently low that the flow is in the molecular regime. In this regime, the mean free path of the molecules is larger than the system size, so that the flow is well described by non-interacting particles moving in straight lines. The particles reflect diffusely, leaving surfaces with a cosine distribution. As in the experiment, the side walls of the box pump the particles. The corrugated panel structure, which increases the surface area of the pumps, is not resolved in the simulation; an increased effective sticking coefficient is used instead. In these calculations, the effect of beam heating of the background gas is neglected. The torus is treated as a perfect pump.

The pumping speed of a sticky surface is given by:

$$
S_{w}=f_{s c} A_{w} \frac{1}{4} \sqrt{\frac{8 k_{B} T}{\pi m}}
$$

where $f_{s c}$ is the sticking coefficient, and $A_{w}$ is the surface area. A perfectly sticky surface of $1 \mathrm{~m}^{2}$ pumps deuterium molecules at $300 \mathrm{~K}$ with a speed of $314 \mathrm{~m}^{3} \mathrm{~s}^{-1}$, for hydrogen molecules it is a factor $\sqrt{2}$ higher. This results in a theoretical maximum pumping speed for $\mathrm{D}_{2}$ of 9.9 . $10^{3} \mathrm{~m}^{3} \mathrm{~s}^{-1}$ if the complete side walls of the NBI box were an ideal pump. An effective sticking fraction of 0.30 is thus needed to reproduce the maximum experimental pumping speed of $3.0 \cdot 10^{3} \mathrm{~m}^{3} \mathrm{~s}^{-1}$. The turbo pumps are implemented as flanges with a sticking fraction of 0.18 to match the pumping speed specified by the manufacturer [8].

Literature sticking coefficients for hydrogen molecules on titanium films are available, but the values range from 0.007 to 0.09 at non-cryogenic temperatures, depending on the film thickness and roughness [9-11]. For deuterium molecules, the sticking coefficients range 
from 0.08 to 0.15 depending on the film thickness [10]. The upper end of the range between hydrogen and deuterium differs by about a factor $\sqrt{2}$, which would cancel out the flux difference. The effective sticking fraction of 0.30 needed in the simulation to reproduce the pumping speed is higher than the literature values since the surface area of the pumps is much larger in the experiment due to the corrugated panel structure. The panel structure increases the surface area by a factor between 2 and 30, based on the extremes of the literature sticking coefficients.

The ion extraction code IBSimu is used for the particle tracking [12]. The surface collisions and diffuse reflections are handled by an additional routine specially written for this purpose. Geometry components are defined as surfaces, read from STL files. This format describes triangular cells by specifying the vertices and surface normals, and can be generated from 3D models by all major CAD software packages. Intersections of particle paths with surfaces are determined with the modified BSP tree algorithm implemented in the Visualization Toolkit [13, 14].

\subsection{Magnetic field calculation}

The Finite Element model for ASDEX Upgrade magnetic field calculations was extended by including NBI box 2 [15]. The magnetic field is a combination of the partially shielded stray field of ASDEX Upgrade, and the deflection field inside the box. The magnetic field is linearly interpolated onto a regular grid by means of the 3D Delaunay triangulation available in the CGAL library [16].

\subsection{Beam propagation}

Because of the aperture structure of extraction grids, the neutral beam consists of a collection of beamlets. In the initialization of the particle ensemble, it is assumed that each beamlet has a Gaussian power density distribution. The beamlets are parametrized by the divergence $\varepsilon$, which is half the opening angle of a cone with power densities that are $1 / \mathrm{e}$ of the on-axis power density. The normalized power density for a single beamlet as function of parallel $\left(1_{\|}\right)$and perpendicular $\left(1_{\perp}\right)$ distance with respect to the beamlet axis is

$$
F\left(l_{\|}, l_{\perp}\right)=\frac{1}{2 \pi \sigma_{\perp}^{2}} \exp \left(-\frac{l_{\perp}^{2}}{2 \sigma_{\perp}^{2}}\right)
$$

with

$$
\sigma_{\perp}=\frac{l_{\|} \tan (\varepsilon)}{\sqrt{2}}
$$

The divergence of $0.8^{\circ}$ and beamlet offset steering of $1.44 \% \mathrm{~mm}$ are obtained from beam emission spectroscopy measurements [2]. The current fractions of extracted $\mathrm{D}^{+}, \mathrm{D}_{2}^{+}$, and $\mathrm{D}_{3}^{+}$ions were previously determined [17]. The resulting particle ensemble at the neutralizer exit was calculated by numerically integrating the differential equations that describe the species evolution in the neutralizer. Background gas interaction after the neutralizer is taken into account with a Monte-Carlo scheme, with cross sections taken from the literature [18, 19]. Heat loads, calculated from the particle flux arriving at the surfaces, are visualized with the Visualization Toolkit [14].

\section{Results}

\subsection{Neutral gas densities}

For a typical ASDEX Upgrade discharge with all 4 sources in NBI box 2 switched on, the measured pressure in the first pumping chamber is $52 \pm 6 \cdot 10^{-6}$ mbar. At a TGP pumping speed of $3.0 \cdot 10^{3} \mathrm{~m}^{3}$ per second, the simulated pressure at the same location is $44 \cdot 10^{-6} \mathrm{mbar}$, which indicates that the titanium getter pumps are operating close to their maximum. At this pumping speed the TGPs remove $99 \%$ of the particles, the rest is pumped by the duct and the turbo pumps. A TGP pumping speed decrease of approximately 10 percent in the simulation results in the measured pressure. Given that the pumping speed in the experiment decreases quickly due to saturation of the surface, the agreement between measurement and calculation is good [7].

Figure 3 shows the density profile along the beam axis, averaged over the four sources, at the ideal TGP pumping speed of $3.0 \cdot 10^{3} \mathrm{~m}^{3}$ per second. The gas flow in the neutralizer was modeled in a separate simulation, to improve the signal to noise ratio in volumes with a low density. The effect of the tapered neutralizer and two chamber structure of the NBI box is clearly visible in the density profile. In the duct region, the density is approximately $1.2 \cdot 10^{18} \mathrm{~m}^{-3}$, which leads to a neutral beam power loss of $1.7 \%$ per meter for the $93 \mathrm{keV}$ beam, taking the distribution over the energy components into account. The density is close to the value of $10^{18} \mathrm{~m}^{-3}$ simulated for the ITER DNB duct [20].

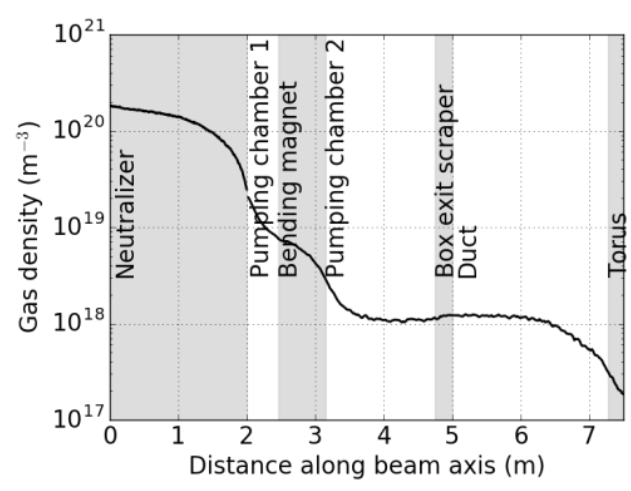

Fig. 3. Calculated gas density profile on the beam axis.

\subsection{Residual ion dump power loads}

To benchmark the magnetic field calculation, the calculated heat deposition on the ion dump panels was compared to the energy deposition in the experiment. Figure 4 shows the calculated profiles for the different sources during discharge 29029 together with the measured profiles. The measured profiles are scaled, and averaged over a few hundred discharges. There is no 
data shown for panel 1 and 6 of source 8, since those thermocouples are broken. The general shape of the calculated profiles agrees well with the measured ones, but all the measured profiles are slightly broader. This might be due to the uncertainty in the divergence, or due to space charge expansion of the beam, which is not included in the simulation.

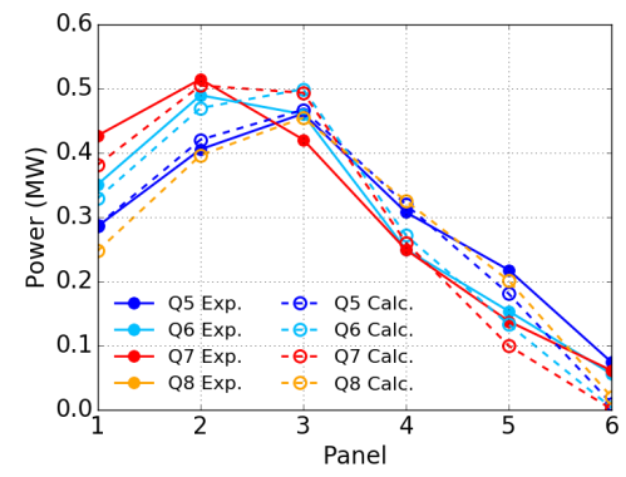

Fig. 4. Calculated and experimental heat deposition on the residual ion dump panels.

\subsection{Transmission losses in the beam duct}

Some transmission losses in the beam duct are unavoidable, geometrical losses due to scraping of the beam on apertures and reionization losses due to interaction of the beam with the background gas. In the ASDEX Upgrade beamline of box 2, sensitive vacuum components have been damaged erratically in locations that were shown to have no direct line of sight to the sources [2]. Figure 5 shows the power losses after the neutralizer as function of the distance along the box axis. The losses are normalized to the total power per species.

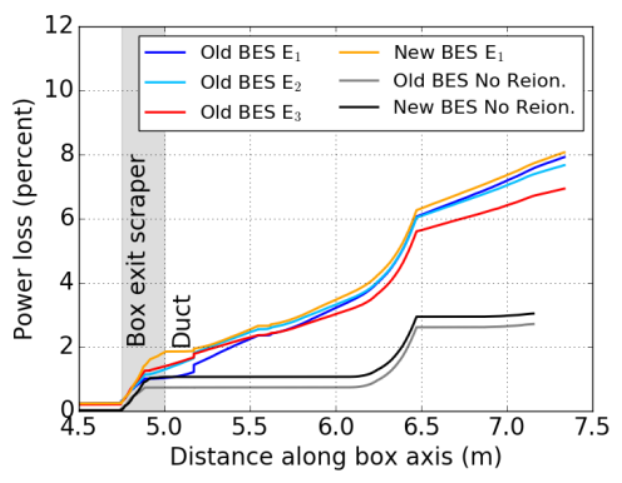

Fig. 5. Losses for source 5 along the beamline with the old and new box exit scraper, for different energy components, with and without reionisation.

Without reionization, the losses are modest, and only occur where duct components scrape the beam. When including reionization, the losses are slightly different for the three energy components because of the larger cross section for ionization at higher energies. The energy deposition profile also changes; a consequential amount of power is deposited in shadowed locations. The power losses in the duct are foreseen in the design, and are dumped on actively cooled components. However, a substantial amount of power is lost at $5.2 \mathrm{~m}$, which is the position of the gate valve, where damage has occurred in the experiment. The power deposition profile for the new BES shows less power deposition in this critical area. The overall transmission for the old and new BES is almost the same. These calculations show that the new BES better protects the sensitive components, while the influence on the overall transmission is minimal.

\section{Conclusion}

A suite of modeling tools was developed to study the gas density, magnetic field, and power deposition for a given NBI beamline. With these tools, injector 2 of the ASDEX Upgrade NBI system was studied. Gas pressures modeled with molecular flow calculations match well with measured pressures, and indicate that the titanium getter pumps are operating within $10 \%$ of their maximum pumping speed. Finite Element calculations were performed to model the magnetic field in the beamline. Particles were tracked through the magnetic field while taking into account background gas interaction. As a benchmark, calculated residual ion dump profiles were compared with calorimetric measurements, good agreement was found. Beam losses were calculated. When no ionization is taking place, there is no power deposition on the previously damaged locations. With reionization, power is deposited at the gate valve location. The new BES protects this location better while preserving the overall transmission.

Modeling of the ASDEX Upgrade beamline shows the usefulness of these tools, but their application is by no means limited to this beamline. The Wendelstein 7-X beamline as well as several test stands at IPP have been studied. New geometries are easily imported from CAD generated STL files. With these tools, future beamlines will be studied and optimized in terms of duct losses.

\section{Acknowledgments}

This work has been carried out within the framework of the EUROfusion Consortium and has received funding from the Euratom research and training programme 2014-2018 under grant agreement No 633053. The views and opinions expressed herein do not necessarily reflect those of the European Commission.

\section{References}

[1] IAEA, ITER Technical Basis, Vienna 2002

[2] N. den Harder et al., EPS Conf. Proc. 41F (2017)

[3] B. Streibl et al., Fus. Sci. Tech. 44 (3) (2003) 578-592

[4] P. Franzen et al., Fusion Eng. Des. 5657 (2001) 487-491

[5] W.L. Wiese et al., Journal of Physical and Chemical Reference Data 38 (3) (2009) 565-720

[6] J.H. Feist et al., Fus. Tech. 1 (1993) 486-490

[7] G. Orozco et al., Fusion Eng. Des. 123 (2017) 381-384

[8] Pfeiffer Vacuum, Operating instructions for Turbomolecular Pumps TPH/TPU 2200, Vienna 2002 
[9] D.J. Harra et al., J. Vac. Sci. Technol 13 (1) (1976) 471474

[10] G. Grigorov et al., Vacuum 34 (5) (1984) 513-517

[11] L. Zeng-rui et al., Vacuum 34 (8) (1984) 747-748

[12] T. Kalvas et al., Rev. Sci. Instrum 81 (2) (2010) $02 B 703$

[13] A. Formella et al., The Visual Computer 11 (9) (1995) 465476

[14] W. Schroeder et al., Visualization Toolkit: An ObjectOriented Approach to 3D Graphics, Kitware 2006

[15] I. Zammuto et al., Fusion Eng. Des. 98 (2015) 1419-1422

[16] C Jamin et al., CGAL User and Reference Manual Edition 4.11.1 2018

[17] P. Franzen et al., Tech. Rep. IPP 4/278 (1998)

[18] A. Stäbler et al., Fus. Tech. 26 (2) (1994) 145-152

[19] T. Tabata et al., At. Data Nucl. Data Tables 76 (1) (2000) 1-25

[20] M.J. Singh et al., AIP Conf. Proc. 1655 (1) (2015) 050011 